\title{
Epistemic game for answer making in learning about hydrostatics
}

\author{
Ying Chen, Paul W. Irving, and Eleanor C. Sayre \\ Department of Physics, Kansas State University, Manhattan, Kansas 66506, USA
}

(Received 12 November 2012; published 14 March 2013)

\begin{abstract}
Previous research into problem solving in physics resulted in researchers introducing six epistemic games to describe the organizational structures of locally coherent resources. We present a new epistemic game- the "answer-making epistemic game" - which was identified in this paper through the analysis of interviews carried out to validate a survey focusing on students' understanding of Archimedes' principle and Pascal's law. In the game, the ultimate goal is a solution to a problem posed by the survey. Students may remember or intuit an answer, then use conceptual and/or mathematical reasoning to justify it. Alternately, they may use conceptual and/or mathematical reasoning to generate an answer. We demonstrate how students generate their solutions using these two different paths and discuss some implications for instruction.
\end{abstract}

DOI: 10.1103/PhysRevSTPER.9.010108

\section{INTRODUCTION}

The idea of epistemic games (e-games) was first introduced as a set of rules and strategies that guide inquiry, focusing on expert scientific inquiry across disciplines [1]. Tuminaro and Redish [2,3] subsequently generalized e-games to be descriptive rather than normative, and generalized the theory to include introductory physics students as well as experts. They define an epistemic game as: "A coherent activity that uses particular kinds of knowledge and processes associated with that knowledge to create knowledge or solve a problem."

Each epistemic game has both ontological components and structural components. The ontological components are the knowledge base and the epistemic form. The knowledge base is all the resources [4] students draw on when they are playing an epistemic game. The epistemic form is the structure (often referred to as the target structure) which helps guide the activity. For example, in a physics problem solving e-game, an epistemic form could be a series of steps that guides the progression in the problem-solving activity. Simultaneously, the structural components include the entry and exit conditions for the game as well as the moves made during the game. The entry and exit conditions (which are determined by the students participating in the game) decide the starting and ending points of each epistemic game. All steps between the starting and ending points of an epistemic game are considered moves which help progress a particular game to completion.

In university introductory classes, students often assess their perception of what the learning environment is asking

Published by the American Physical Society under the terms of the Creative Commons Attribution 3.0 License. Further distribution of this work must maintain attribution to the author(s) and the published article's title, journal citation, and DOI.
PACS numbers: 01.40. $-\mathrm{d}$, 01.55.+b, 01.40.Ha, 01.40.G-

of them and then frame their activities appropriately. Framing is an active bringing together of several resources in an effort to understand and work within a situation [5]. In contrast to framing, e-framing is a perception (unconscious or conscious) of what tools and skills are needed in a particular context or situation. E-frames can be envisioned as a storage area for conceptual and procedural resources, promoting some resources activation and blocking others. An individual's framing consists of many components, but Tuminaro uses the skills and epistemological components of an individual's framing to categorize e-games [3]. When students frame an activity as needing a certain set of skills and involving a certain type of knowledge, then they may enter into a particular e-game. For example, the "Recursive Plug-and-Chug" e-game identified by Tuminaro [3] is one in which students plug quantities into physics equations in order to achieve a numerical answer without ever understanding the conceptual understanding underlying their calculations. University introductory classes produce the opportunity to enter into this type of e-game. Another common occurrence in an introductory physics class is completing the worksheet and getting the answers, in which students operate in a frame known as "answer making" [6]. This is different from a sense-making frame in which students frame their activities as making sense of the world.

In this paper, we introduce a new e-game: the "answermaking epistemic game" (AMEG). This e-game was discovered while members of the research group were in the process of validating a task that was to be used to evaluate students' learning of Archimedes' principle and Pascal's law post hydrostatics instruction. The task consisted of several questions, the majority of which were multiple choice. On review we found that students were engaging in the AMEG when they were answer making on the multiple-choice conceptual questions which were very similar in design to those found on common conceptual 
surveys like the Force Concept Inventory [7] or Conceptual Survey of Electricity and Magnetism [8]. It is our intention to highlight that this is an e-game that we have observed within this context of a validation interview of multiplechoice questions on hydrostatics that may be transferable to other contexts. Its applicability to other contexts is not addressed in this paper, however, we believe it is plausible that students engage in this game in other contexts. In this paper we focus on the properties of the AMEG as seen in the hydrostatics validation data; our intent is also not to expand on the literature on students' ideas in hydrostatics.

Previous research on hydrostatics $[9,10]$ has indicated that students have difficulties with hydrostatics concepts such as Archimedes' principle. It has also indicated that students have difficulty explaining the sinking and floating behavior of objects and are unable to identify the forces exerted on an object by a fluid. In the past, research has indicated that students have a tendency to make ex post facto explanations to justify an intuition they have about a hydrostatics principle, which makes it an interesting topic in which to explore answer making.

\section{STUDY DESIGN}

The data in this paper are drawn from clinical interviews with students enrolled in an introductory calculus-based physics class taken primarily by engineering majors. The course consists of two one-hour lectures and two two-hour combined recitation laboratories called "studios" each week for 15 weeks. Homework for this course uses an online homework system (MasteringPhysics) which encourages students to submit correct answers and cannot grade them on their problem-solving process. At the time of the interviews, the class had recently covered hydrostatics for 2.5 weeks in lecture and studio.

The thrust of the interviews was to validate a short task on Archimedes' principle and Pascal's law; thus, the protocol asked students to solve ten related problems in multiple-choice or free-response format. Students were instructed to think aloud as they solved problems, and encouraged to share their reasoning as well as their answers. Though they had recently covered hydrostatics in class, our interviews were the students' first exposure to our questions: we did not release questions ahead of time to students, and the questions were not drawn directly from their course work. Conversely, the problems on the hydrostatics assessment were similar to canonical ones in hydrostatics, and "fair game" for students in this course.

Initially, we were not expecting to see students produce answers followed by justification; rather, we expected reasoning followed by answers. This violation of our expectations for students' problem-solving strategies prompted us to examine the games students play in more detail. We observed that five students consistently behaved similarly in the interviews, using the same kind of strategies to answer the questions. We infer that they use similar mental processes and frame the activity similarly. All of the data collected from their clinical interviews fits within the AMEG, as we will show in the next section.

\section{ANSWER-MAKING EPISTEMIC GAME}

Answer making, as an epistemic frame, is marked by student behaviors like worksheet completion and getting the right answer. It sits in contrast to sense making, in which students try to figure out phenomena about the physical world. Behaviors associated with sense making include making connections to the real world or lived experience, coordinating multiple representations, considering the reasonableness of solutions, and treating the problem as a sensible one to solve. Within that framing, we note that students regularly perform the same series of moves in solving problems, detailed in Fig. 1.

\section{A. Structure}

Within the AMEG, the ultimate goal is to produce an answer to the worksheet problem together with some (perhaps minimal) justification or reasoning for the answer (the "sufficient solution"). The entry condition for the AMEG is students asking themselves if they remember the answer to the problem, and (if not) if they can quickly intuit an answer. If they can either remember or intuit an answer, they embark on the answer leads to justification (AJ) path. If not, they embark on the reasoning leads to answer (RA) path. The structure of the game is shown in Fig. 1.

The two paths differ substantially in the role that the answer to the question plays. In the AJ path, the answer occurs first. The justification reassures the student that her previously chosen answer is correct. In contrast, on the RA path, the student's reasoning leads her to an answer. Both paths reach the same end condition of a sufficient solution, but the answers originate in two different places: the RA path to the sufficient solution is based firmly in a more rigorous reasoning process than the AJ path. The distinction between the AJ and RA paths opens up the possibility that students will try to justify answers that are different from the ones they would arrive at through reasoning [11].

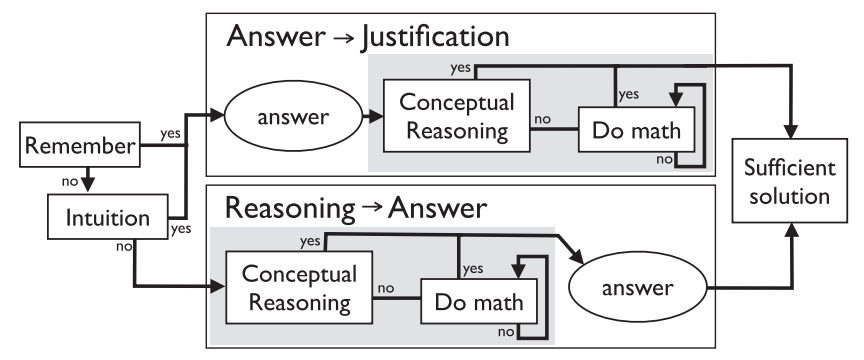

FIG. 1. The answer-making e-game. Upper box: Answer leads to Justification (AJ) path. Lower box: Reasoning leads to Answer (RA) path. Shading indicates the justification and reasoning moves. 
When we examine the kinds of reasoning or justification on both paths, we notice that students first engage in conceptual reasoning to justify the remembered or intuited answer (AJ path) or to generate a new one (RA path). If the student feels the conceptual reasoning is sufficient and complete, he either uses it to generate an answer (RA path) or to justify his preexisting remembered or intuited answer (AJ path), and exits the game with a sufficient solution.

If the conceptual reasoning fails-either because the student feels confused or because he cannot remember the concepts-he turns to mathematics. It is important to note that students make their own judgments as to whether or not conceptual reasoning has failed them: the original intent of the interviews was to validate a brief hydrostatics assessment, not to teach students or uncover new ideas about students' understanding of the subject material, and so the interviewer did not press students about the correctness or completeness of their ideas.

Should students turn to "do math," their initial math attempts are usually not very rigorous: they do not start from first principles, they do not distinguish between densities of different substances (with subscripts, for example), and/or they may leave out some terms altogether. If the initial math does not yield an answer, students engage in more rigorous or elaborate math. This can end up in a circular pattern of many iterations of doing more math until a sufficient justification (AJ path) or reasoning (RA path) is reached. As with the conceptual step, students make their own judgments about whether their justification or reasoning is sufficient and satisfying.

These moves are based on the analysis of five students answering multiple questions with each individual student often using different moves in different questions. Each student engaged in both paths of the AMEG, and we find that features of this game are consistent across multiple problems and multiple students. It is possible that a more extensive data set could uncover additional moves, such as what happens when do math fails. In the next section, we present two examples of students using the game.

\section{B. Ontology}

In regards to the ontological components, the knowledge base for the three moves of "remember," "intuition," and "conceptual reasoning" are generated from the students' formal physics resources and common sense resources. Students often rely on their own knowledge of physics they have developed and not on direct reference to physics principles. However, the knowledge base shifts when students are confronted by problems in which it is more arduous to construct a justifiable answer. This was often the case in problems we think are harder. Students had to resort to several moves of "do some math," eliciting more formal physics and mathematics resources.
The epistemic form of this game could be described as a truth table. The structure and rules are based on a "yes" and "no" protocol which in the latter parts of the e-game combines with the mathematical expressions that students write down during the process of problem solving. This is in accordance with descriptions of epistemic forms as the squares that are filled out in tic-tac-toe, or the list for a list making game [3].

\section{A STUDENT PLAYS THE AMEG}

We will present a case study of a student which demonstrates how students play the AMEG. The case study illustrates examples of both possible paths for the AMEG indicated in Fig. 1 and highlights what we observed from all of the interviews: a singular student will take different paths dependent on the relative difficulty of the problem and which resources they activate. We divide the discussion into three examples which correspond to different options of this game. The student demonstrates several different moves in two different problems. "Remember the result" is covered by one problem about water levels in three different containers that are differently shaped but all connected together. Intuition, conceptual reasoning, and do some math are covered by the boy in the tub problem illustrated in Fig. 2.

\section{A. Remember the result}

Students often believe what they have previously observed and experienced to be right, and accept conceptions derived from these experiences without difficulty. Because of these conceptions being built upon what has been observed and experienced students often do not build an explanation or justification for the concepts until required. In this context it was observed that students will remember the answer and then in order to make sense of their answer they will attempt to build a justification for it.

A child is playing with toys in the bathtub, including a large toy boat with various crew members. You notice that the water level in the tub suddenly gets slightly

lower. Which of the following actions by the child could have resulted in that change in water level? Choose all which apply

A The child dropped a crew member from the boat into the tub, and the crew member sank to the bottom of the tub.

B The child dropped a crew member from the boat into the tub, but the crew member floated in the water.

C The child threw a crew member from the boat on to the floor outside of the tub.

D None of the listed options would cause the water level in the tub to become lower.

FIG. 2. The boy and tub problem. 
In this example the student is quite certain that he remembers the answer from a similar experiment he had previously conducted, but his memory does not extend to the reasoning as to why the answer is as he remembers. The context of this problem is the previously discussed scenario of asking the student what the final water level will be of three containers of water of different shapes that are all connected to each other.

Student: My first thought, when I think of this problem, is back in high school physics and physics class I am taking right now. We always did the experiment with some crazy shaped containers; where each of the different containers for water was different, but they were all connected underneath. And the water level was always the same. Because, ah, the actual reason I cannot completely remember, but I do know that's the answer.

Interviewer: So you reason like that because saw that, it's true, right?

Student: Ah, that's my, ah, that's my first thought...yes, I should know the actual reason, hmm...I am pretty sure, because...err...the pressure is equal on the top of each of these, but there is a pressure difference... I just know the shape of the container does not matter.

When prompted, he constructs a postfact justification for his answer. In this context the student perceives that he cannot just provide the answer and instead must provide a justification for his answer. His justification is minimal, but by mentioning that his answer is something to do with pressure is enough justification to meet the required end condition and so exits the AMEG quickly, repeating that "I just know the shape of the container doesn't matter."

\section{B. Intuition and conceptual reasoning}

The same student as above plays the answer-making e-game in the following problem (Fig. 2). This time the student uses several iterations of AMEG because the problem is being a multiple-choice multiple response question and he considers each choice separately. The student starts at the entry condition of remember, but quickly indicates that he cannot remember any prior result for any of the choices.

He then returns to A and chooses an intuitive answer: the water level will not change if the crew member stays in the tub (thus, by that assertion answers A and B are correct). Without calculation or much thinking, or any clue from memory as that in the remember move, the student made his conclusion at once. This is what we called the intuition move (sometimes the students would say "based on intuition, the answer should be..."). Then he justifies his choice as follows:
Student: Obviously, if one little crew member comes out of the boat, the water level, or the boat rise a little bit in the water, and it would increase the, or decrease the water level in the bath tub. But that same figure's been placed in the water, so it's making up for the amount that, or the change on the, where is it, the water level of that the boat does. So these would have...no net change I believe.

For completeness, the student then formally evaluates each given answer choice, starting with $\mathrm{C}$, followed by $\mathrm{B}$. When he continues to specifically evaluate answer C (the crew member leaves the tub), he says that "I know for sure that $\mathrm{C}$ is true, because as I've explained earlier, if the boat weight is less it's gonna displace less, or it's gonna float higher in the water, and displace less fluid, so, therefore, the water level would drop a little bit. So C is definitely right."

\section{Do some math}

When the student evaluates answer B, he starts with the kind of intuitive reasoning that allowed him to evaluate $\mathrm{C}$. However, he confuses himself and cannot come to an answer:

Student: B, I'm not sure, because I'm trying to think, whether or not, the amount of the water, the amount of the displacement that the, or the change of the displacement of the boat would be equal to the change of the displacement of the person being added to the water. If those two displacements are equal, then yes; or then no, it doesn't change the water level.

He stops talking, and starts to write down all equations relating buoyancy to weight. After that, he decides that the buoyancy force depends on the volume displaced. He was not completely sure about answer B being correct and thinks about this for a minute, at the end of which he states confidently, "Yeah, I believe this one [B] doesn't change the water level in the bath tub." He justifies his answer with mathematics, writing down several formulas and referring to them.

His extended work with answer choice B is replicated when he returns to work with answer A: conceptual reasoning which fails to yield an answer followed by fruitful mathematics.

\section{RELATIONSHIP TO OTHER E-GAMES}

Some moves in our e-game have some obvious similarities to the e-games identified by Tuminaro and Redish [2]. In particular, Physical Mechanism, Mapping Meaning to Mathematics, Mapping Mathematics to Meaning, and Pictorial Analysis are also closely related to the AMEG. We contend that the AMEG is of a larger scale than any of 
those games, and may help students coordinate choices between which subgame to play.

The presence of the intuition move and then building justification to exiting holds the similar ontological components as their Physical Mechanism game. We distinguish the AMEG from Physical Mechanism in two ways: the AMEG has several more possible moves; in the Physical Mechanism game, the end condition requires students to evaluate the conceptual story that they developed. In the AMEG, students are often not trying to tell a conceptual story but instead are trying to build a justification for either their remembered or intuitive answer. The AMEG also differs in entry conditions from the Physical Mechanism game, whose sole entry condition is an intuitive belief about a physical mechanism. In the AMEG, one can start with a remembered answer or an intuitive belief, or use conceptual reasoning or mathematics to generate an answer. Constantly seeking a sufficient solution may lead students to math and equations, components which are not covered by the Physical Mechanism game.

The broader scope of the AMEG does not preclude students from playing the Physical Mechanism game. In all of our interviews, no student got the right answer based solely on intuition, though several wrong answers resulted (this may be a result of the problem being uncommon in daily life). The lack of answer after consulting both intuition and memory results in students traversing the RA path. It is possible that, had students more experience with these problems, they could have traversed the AJ path more frequently because of their intuitions (instead of their memories), which might have resulted in students playing the Physical Mechanism game.

Both the Mapping Meaning to Mathematics game and the Mapping Mathematics to Meaning game are contained in the same way that the Physical Mechanism game was contained within the AMEG if certain choices are made at certain points. There are similarities between the conceptual reasoning and do math section of the game that have obvious similarities to the Mapping Meaning to Mathematics game, or in the opposite direction, the same comparison between do some math conceptual reasoning and mapping mathematics to meaning. In this case again they both have the same ontological components. In both of these cases, students try to imagine the physical situation, and express their thoughts about it through math. Or they start with a physics equation and then attempt to relate physical meaning to that.

Finally, in regard to comparisons between the AMEG and previous e-games, in our interviews students consistently drew diagrams when they were trying to give a conceptual explanation. During the interviews the students drew free-body diagrams or a physical picture to help them understand the physical situation that the problem was describing. This is considered to be a tool students usually employ when doing conceptual reasoning. However, it can also be related to a previously identified e-game, which is called Pictorial Analysis by Tuminaro and Redish.

The primary difference between AMEG and other previous games is the scope of the game: the AMEG includes the option to enter into four previous e-games: Mapping Meaning to Mathematics, Mapping Mathematics to Meaning, Physical Mechanism game, and Pictorial Analysis game during play. Each one of these games has the possibility of occurring during the AMEG, but by themselves they could not account for the complex process of the students solving these problems in this context. For this reason, we think of the AMEG as being like a supergame which governs the order of which other games students might play in the process of answer making.

\section{DISCUSSION AND IMPLICATIONS}

\section{A. Difference between students}

We found that different students will play this game differently in different problems, often choosing different paths through the game depending on the resources available to them. In general, two major points significantly affect students' reasoning and the moves they choose to take. First, the problem they are working on can influence students' thinking. If students think the problem is simple and the answer is "easy" to remember, they state their answer quickly. However, their level of confidence is dependent on being able to remember or construct some justification for their answer. If they cannot remember the answer or intuit one quickly, they will activate reasoning resources, which will decide the branches and hence moves they take.

Even though our questions are conceptual, many students attempt to solve them using algebra, so it is interesting to touch on how students use math here. There is substantial variation in their algebra skills: some students try to avoid math altogether, spending more time with conceptual proportional reasoning (for example, as with Ohm's p-prim [12]) without resorting to more formal algebra. Other students progress more quickly through the RA branch and use equations, subscripts, and other trappings of formal algebra more readily. For complicated questions, students of both kinds eventually have to use equations. Based on our data, when students tend toward many iterations of the do math move they often become more confused and doubtful about their result the more math they do.

\section{B. Implications}

The AMEG lends important insight into how students solve conceptual problems. It covers four of the previous e-games which all include the element of conceptual reasoning, describing students' thinking processes which students apply during their attempts at seeking a solution to 
problems. The fact that these students played this game so readily when faced with multiple-choice and short-answer physics questions suggests that they play this game commonly and that it is not unique to the interview setting or to hydrostatics.

At its heart, it acknowledges that the students' goal in solving these problems is not (necessarily) displaying a robust conceptual understanding, nor is it to reason through the scenarios based on rigorous reasoning. The students' goal is clear: answer the questions with minimal time or effort. This clarity of goal may change depending on the context with which the student is confronted. For example, in a test setting the goal may be to "answer the questions right in as much time as is allowed." If investigating the use of the AMEG in a different setting, this needs to be considered. This answer-making frame leads students through a succession of choices, which may be different from what instructors or test designers desire.

For example, a common instructional gambit (in tutorials [13], for example, though widely used elsewhere) is to have students answer a conceptual question and "explain [their] reasoning." Tutorial instructors sometimes have difficulties encouraging students to adequately explain their reasoning. We can describe the students' strategy using the AMEG. Answer-making students prefer producing an answer and then justifying it (the AJ branch) to performing some reasoning which leads to an answer (the RA branch). The question order in the tutorial further encourages them to follow this path (answer first, then explain reasoning). It is possible that reframing students' involvement with tutorials (through curricular changes or TA training, for example) will reduce students' use of the AMEG, but proposing and testing those changes is beyond the scope of this paper.

\section{Hydrostatics}

In addition to the work on e-games, this project also has implications for student understanding of hydrostatics. Students answering questions in hydrostatics seem to have a predilection for answering questions with intuitive leaps. For example, Loverude describes students' explanations of a problem involving buoyancy of five blocks of different mass as "It is possible that many explanations were attempts to justify a prediction based on intuition, rather than attempts to articulate a line of reasoning that leads to a prediction." [9].

The AMEG gives an insight into the workings of this intuition justification strategy. In regard to specific concepts, our interviews indicate that students believe that water level does not change as long as conditions in a container remain the same (no matter where the objects stay in the water). Students applying the AMEG to this type of problem consistently used this as a starting intuition which they then tried to justify through conceptual reasoning. It remained durable even through reasoning and justification.

Evidence from this study indicates that introductory level students have a vague idea about Pascal's principle, which they do not apply consistently in a correct manner. For example, most of them express that the pressure should be equal at the same height from water level. However, they inconsistently apply this belief when there are some variations in the problem scenario, such as the different shaped containers or different amount of fluid beneath the point being considered.

This paper is part of a larger study of student thinking across many introductory physics topics [14-16], and the data discussed here are taken from validation interviews for an instrument to test students' understanding of Archimedes' principle and Pascal's law. While our claims are grounded in these topics, we believe the AMEG is not unique to them. Further research is necessary.

\section{ACKNOWLEDGMENTS}

The authors are grateful to D. J. Wagner for the longer version of the survey on which these problems were based. This research is partially supported by NSF CCLI Grant No. 1240782.
[1] A. Collins and W. Ferguson, Epistemic forms and epistemic games: Structures and strategies to guide inquiry, Educ. Psychol. 28, 25 (1993).

[2] J. Tuminaro and E.F. Redish, Elements of a cognitive model of physics problem solving: Epistemic games, Phys. Rev. ST Phys. Educ. Res. 3, 020101 (2007).

[3] J. Tuminaro, "A cognitive framework for analyzing and describing introductory students' use and understanding of mathematics in physics," Ph.D. dissertation, University of Maryland, 2004 (unpublished), available at http:// www.physics.umd.edu/perg/dissertations/tuminaro/.
[4] D. Hammer, Student resources for learning introductory physics, Am. J. Phys. (Phys. Ed. Res. Suppl.) 68, S52 (2000).

[5] E. C. Sayre and M. C. Wittmann, Plasticity of intermediate mechanics students' coordinate system choice, Phys. Rev. ST Phys. Educ. Res. 4, 020105 (2008).

[6] R. E. Scherr and D. Hammer, Student behavior and epistemological framing: Examples from collaborative activelearning activities in physics, Cognit. Instr. 27, 147 (2009).

[7] I. A. Halloun and D. Hestenes, The initial knowledge state of college physics students, Am. J. Phys. 53, 1043 (1985). 
[8] D. P. Maloney, T. L. O’Kuma, C. J. Hieggelke, and A. V. Heuvelen, Surveying students' conceptual knowledge of electricity and magnetism, Am. J. Phys. 69, S12 (2001).

[9] M. E. Loverude, C. H. Kautz, and P. R. L. Heron, Helping students develop an understanding of Archimedes' principle. I. Research on student understanding, Am. J. Phys. 71, 1178 (2003).

[10] P. R. Heron, Empirical investigations of learning and teaching, Part I: Examining and interpreting student thinking, in Research on Physics Education, Proceedings of the International School of Physics "Enrico Fermi," Course CLVI, edited by E. F. Redish and M. Vicentini (IOS Press, Amsterdam, 2003), pp. 341-350.

[11] J. Hawkins, B. Frank, J. R. Thompson, M. C. Wittmann, and T.M. Wemyss, Probing student understanding with alternative questioning strategies, in Proceedings of the Physics Education Research Conference, Philadelphia,
2012 (AIP, New York, 2013), http://arxiv.org/abs/ 1107.1825 .

[12] A. A. diSessa, Toward an epistemology of physics, Cognit. Instr. 10, 105 (1993).

[13] L. C. McDermott and P. Shaffer, Tutorials in Introductory Physics (Prentice Hall, Upper Saddle River, NJ, 1998).

[14] E. C. Sayre, S. V. Franklin, S. Dymek, J. Clark, and Y. Sun, Learning, retention, and forgetting of Newton's third law throughout university physics, Phys. Rev. ST Phys. Educ. Res. 8, 010116 (2012).

[15] E. C. Sayre and A. F. Heckler, Peaks and decays of student knowledge in an introductory E\&M course, Phys. Rev. ST Phys. Educ. Res. 5, 013101 (2009).

[16] A. F. Heckler and E. C. Sayre, What happens between preand post-tests: Multiple measurements of student understanding during an introductory physics course, Am. J. Phys. 78, 768 (2010). 